\title{
Active photonic crystals based on surface acoustic waves
}

\author{
M. M. de Lima, Jr., ${ }^{\text {a) }}$ R. Hey, and P. V. Santos \\ Paul-Drude-Institut für Festkörperelektronik, Hausvogteiplatz 5-7, 10117 Berlin, Germany
}

(Received 28 July 2003; accepted 18 August 2003)

\begin{abstract}
An active photonic crystal (PC) based on the modulation of a one-dimensional cavity resonator by electrically-generated surface acoustic waves is described. The high nonthermal population of surface modes combined with the enhanced Brillouin scattering in the cavity increases the intensity of the scattered light to values comparable to the excitation intensity. This process is employed to switch and modulate light beams in PCs. (C) 2003 American Institute of Physics.
\end{abstract}

[DOI: $10.1063 / 1.1617368$ ]

Great attention has been directed towards active photonic crystal (PC) structures, which are able to dynamically control the light wavelength, polarization, or propagation direction using an external stimulus. These structures are technologically important for the realization of tunable optical filters, modulators, and switches. Recently, some degree of tunability has been achieved by infiltrating a photonic crystal containing air holes with a liquid crystal and exploring the strong dependence of the dielectric properties of the liquid crystal on temperature and electric field. ${ }^{1-4}$

In this letter, we propose and demonstrate a concept for active one-dimensional PCs based on the acoustic modulation of a photonic resonator, which consists of a $\lambda / 2$ optical cavity $(C)$ surrounded by Bragg mirrors $\left(\mathrm{BM}_{1}\right.$ and $\left.\mathrm{BM}_{2}\right)$ illustrated in Fig. 1. ${ }^{5}$ The resonance condition is achieved when the vertical component of the light wave vector $k_{z}$ is equal to $k_{c}=4 \pi n_{c} / d_{c}$, where $d_{c}$ and $n_{c}$ denote the thickness and the refractive index of the cavity layer, respectively. For normal incidence, the resonance wavelength becomes $\lambda_{c}$ $=2 \pi / k_{c}$. The modulation by lattice vibration has been previously proposed as a way to achieve active wavelength tunability as well as the control of light propagation direction in PCs. ${ }^{6,7}$ In the present approach, however, the electromagnetic fields become strongly concentrated in the cavity region at the resonance frequency, which significantly increases the scattering of light by lattice vibrations. In fact, a gigantic enhancement of the cross section for Raman and Brillouin scattering in planar cavities have been reported. ${ }^{8,9}$ Here, we demonstrate the dynamic control of light propagation by Rayleigh surface acoustic waves (SAWs) generated by interdigital transducers (IDTs) deposited on top of the layer stack (cf. Fig. 1). The strong acoustic and optical fields in the cavity region enhances the intensity of the Brillouin scattered light to values comparable to the one of the exciting beam. Finally, we show that the SAW-driven resonator acts as a compact Bragg cell with a thickness of less than 5 $\mu \mathrm{m}$, which can be configured to operate as a switch, a frequency shifter, or a modulator.

The optical modulation by the SAW is mediated by three mechanisms. The first comprises the conventional elasto- and electro-optical modifications of the refractive index by the SAW strain and piezoelectric fields, respectively. For wave-

${ }^{a)}$ Electronic mail: mmlimajr@pdi-berlin.de lengths away from electronic transitions, which will be the case discussed here, the elasto-optic contribution $\Delta n_{\text {eo }}$ normally dominates over the electro-optic one. The second mechanism results from the modulation of the cavity thickness $d_{c}$ by the vertical strain component $u_{z z}=\partial u_{z} / \partial z$ of the SAW displacement field $\mathbf{u}$ (cf. Fig. 1). The third is associated with the modulation of the interface profiles by the SAW displacement field, thus creating a structural grating for the incident light.

The optical resonators were designed to operate at optic and acoustic wavelengths of $900<\lambda_{c}<1000 \mathrm{~nm}$ and $\lambda_{\text {SAW }}$ $=5.6 \mu \mathrm{m}$, respectively. In the design, one has to take into account the depth dependence of the SAW strain field. $\mathrm{Nu}-$ merical calculations of this field in GaAs/AlAs BMs and of the resulting elasto-optic contribution $\Delta n_{\mathrm{eo}}$ for light polarized along $y$ yield a maximum modulation amplitude of $\Delta n_{c} /\left(n_{c} \sqrt{P_{l}}\right)=3.9 \times 10^{-5} \sqrt{\mathrm{W}^{-1} \mathrm{~m}}$ for $\lambda_{c}=1000 \mathrm{~nm}$ and depths between $0.25 \lambda_{\mathrm{SAW}}$ and $0.45 \lambda_{\mathrm{SAW}}$. In this expression, $P_{l}$ denotes the acoustic power flux per unit length along the SAW beam cross section. An important feature of the acoustic modulation resides in the fact that the modulation of the thicknesses and of the interface profiles can be substantially larger than the elasto-optic one. Indeed, approximately twothirds of the maximum $\Delta n_{c} / n_{c}$ is accounted for by the strain contribution $u_{z z}$, thus demonstrating its dominating role. The resonators were grown on a GaAs (001) substrate using molecular-beam epitaxy. The cavity depth was selected so as

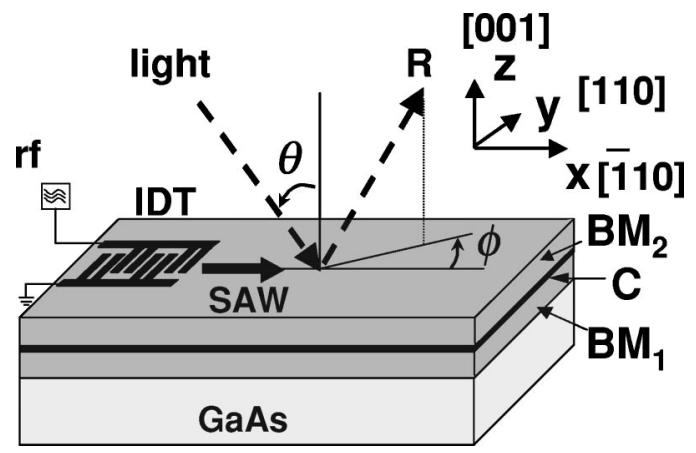

FIG. 1. Optical resonator driven by SAWs, consisting of an optical cavity (C) placed between Bragg mirrors $\left(\mathrm{BM}_{1}\right.$ and $\left.\mathrm{BM}_{2}\right)$. The SAWs are generated by an IDT. The optical experiments are performed by measuring the reflectivity $R$. $\theta$ and $\phi$ denote the polar and the azimuthal angles of incidence, respectively. 


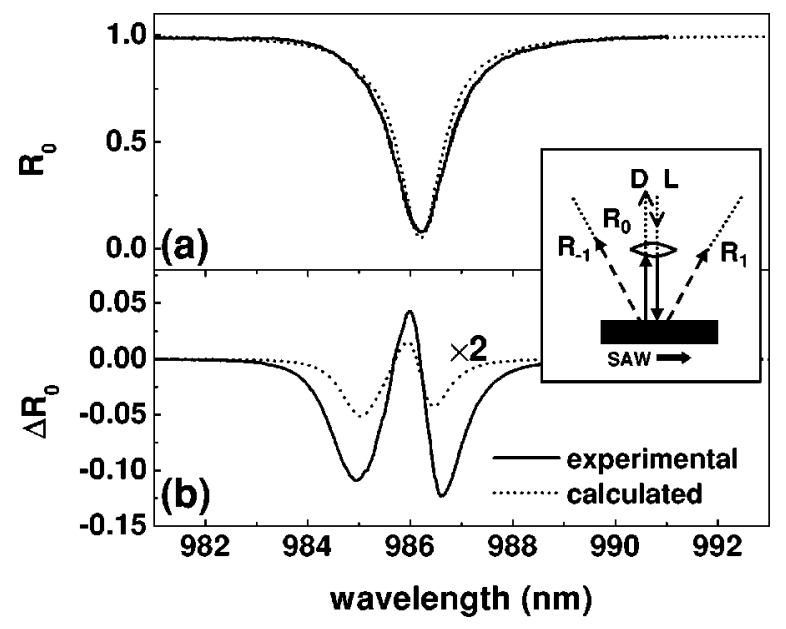

FIG. 2. (a) Reflectivity $R_{0}$ and (b) reflectivity change $\Delta R_{0}$ induced by a SAW with $P_{l}=300 \mathrm{~W} / \mathrm{m}$, recorded in the resonator in the geometry indicated in the inset. $L$ and $D$ denote the light source and detector, respectively, and $R_{m}(m=0, \pm 1, \ldots)$ the $m_{\text {th }}$ diffraction order.

to maximize $\Delta n_{c} / n_{c}$. The lower Bragg mirror $\mathrm{BM}_{1}$ is composed of 15 periods, each containing a $\lambda / 4$ AlAs and a $\lambda / 4$ GaAs layer. The first five periods of the upper mirror $\mathrm{BM}_{2}$ are identical to those in $\mathrm{BM}_{1}$, in the remaining five periods, which are located adjacent to the cavity, the thickness of the GaAs layers was increased to $3 \lambda / 4$. While the larger GaAs thickness does not affect the optical properties, it reduces the effective acoustic velocity near the surface and helps to concentrate the SAW fields in this region.

SAWs propagating along the [110] surface direction were generated by aluminum split-finger IDTs deposited on top of the layer stack. The IDTs were excited by continuous rf power levels of up to $25 \mathrm{~dB}$ provided by a rf generator. The fraction of the applied electrical power converted into a SAW mode by the IDT, however, is only $32 \%$ as determined using a network analyzer. The incident light was polarized perpendicularly to the SAW propagation direction and, unless otherwise specified, focused onto a spot with diameter $\phi_{d}=40 \mu \mathrm{m}$ on the sample surface using an objective with small numerical aperture $(f / 0.055)$, in order to ensure a narrow distribution of the angles of incidence. The absolute reflectivity of the Bragg mirrors was found to be comparable (within $\pm 2 \%$ ) to that of a silver mirror with nominal reflectivity of $98 \%$. We assume, therefore, the reflectivity of the resonator to be unity away from the resonance wavelength.

The reflectivity $R_{0}$ of the resonator under normal incidence is displayed by the solid line in Fig. 2(a). The calculated reflectivity (dotted line) reproduces well the measured quality factor $Q$ of approximately 800 (the calculated wavelengths were slightly scaled to match the measured $\lambda_{c}$ ). The change in the normal reflectivity $\Delta R_{0}$ induced by a SAW with $P_{l}=300 \mathrm{~W} / \mathrm{m}$ is shown in Fig. 2(b). Both $R_{0}$ and $\Delta R_{0}$ were measured on the same spot using a modulation technique in order to correct for variations in sample temperature due to the rf excitation, which may cause shifts in the resonance frequency. $\Delta R_{0}$ is nonzero only within the small range of wavelengths around the cavity resonance. Its spectral shape can be qualitatively understood by taking into account that the SAW modulation shifts the resonance wavelength sinusoidally with an amplitude $\Delta \lambda_{c}$ around the center value Downloaded 08 Oct 2003 to 62.141 .165 .110 . Redistribution subject

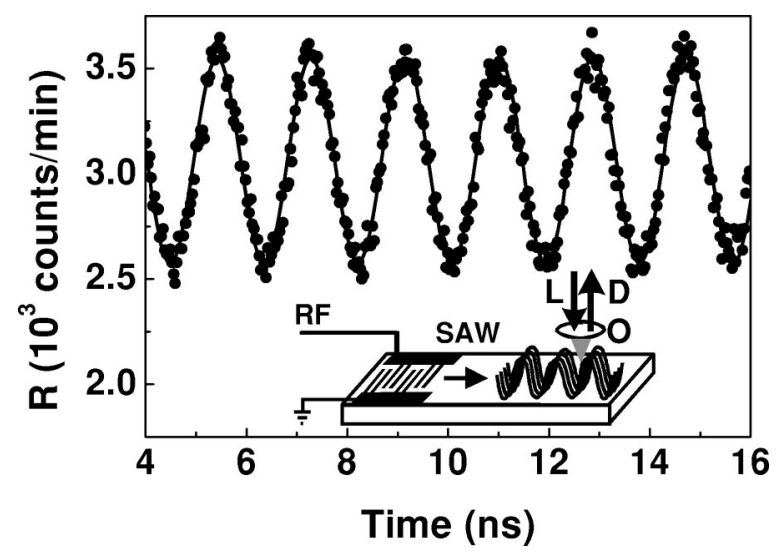

FIG. 3. Time-resolved reflectivity at the wavelength $\lambda_{+f}=986 \mathrm{~nm}$, recorded under a SAW with $P_{l}=150 \mathrm{~W} / \mathrm{m}$ using a $50 \times$ objective $\mathrm{O}$ to focus the light onto a $\phi_{d}=2.6 \mu \mathrm{m}<\lambda_{\mathrm{SAW}} / 2$ spot (inset).

$\lambda_{c}$. As a result, the time-averaged probability $p(\lambda)$ of having the resonance at a particular wavelength $\lambda$ becomes $p(\lambda)=1 /\left[\pi \sqrt{1-\chi^{2}}\right]$, where $|\chi|=\left|\left(\lambda-\lambda_{c}\right) /\left(\Delta \lambda_{c}\right)\right|<1$. In this approximation, the reflectivity change becomes $\Delta R_{0}(\lambda)=\left[p(\lambda) \oplus R_{0}(\lambda)-R_{0}(\lambda)\right]$, where $\oplus$ denotes the convolution operator. The largest reflectivity reduction is thus expected close to the wavelengths $\lambda_{ \pm f}=\lambda_{c} \pm \Delta \lambda_{c}$ at the flanks of the resonance curve. In contrast, the reflectivity at the resonance wavelength $\lambda_{c}$ increases, since the modulation detunes the cavity during both SAW half cycles.

The dotted line in Fig. 2(b) reproduces $\Delta R_{0}$ as calculated using the transfer matrix approach outlined in Ref. 7, which relates the amplitudes of the light field at the frequencies $\omega+m \omega_{\mathrm{SAW}}$ and wave vectors $k+m k_{\mathrm{SAW}}$ in each layer to those in the neighboring layers. The results shown in Fig. 2(b) neglect modes with $m \geqslant 3$. The calculations reproduce reasonably well the spectral shape of $\Delta R_{0}$, but underestimate the modulation amplitude. The main reason for this discordance is the fact that the calculations neglect the modulation of the interface profiles by the SAW fields and do not take into account the finite size of the illumination spot $\left(\phi_{d} \approx 7 \lambda_{\mathrm{SAW}}\right)$ in the experiments, which partially relaxes wave vector conservation.

Focusing the light to a spot with dimensions smaller than half of the SAW wavelength, as indicated in the inset of Fig. 3 allows us to access the time-dependent character of the SAW modulation. The reflectivity (circles in Fig. 3) is modulated at the SAW frequency of $537 \mathrm{MHz}$ with a ratio $\Delta R / R_{d c}$ between the peak amplitude and the average value of $15 \%$. The wavelength dependence of $\Delta R / R_{d c}$ is displayed by the circles in Fig. 4. As in Fig. 2, the modulation amplitude is maximal at the flank wavelengths $\lambda_{+f}$ and $\lambda_{-f}$ and becomes very small at the cavity resonance wavelength $\lambda_{c}$. The phase of the oscillations (squares) at the two sides of the resonance is shifted by $180^{\circ}$ with respect to each other, since the cavity resonance approaches these wavelengths at opposite SAW cycles.

If the resonator is illuminated under a small angle of incidence $\theta$ satisfying $\tan \theta=\lambda_{c} /\left(2 \lambda_{\text {SAW }} \cos \phi\right)$, the wave vector conservation for Bragg diffraction is ensured with the first-order diffraction beam (indicated by $k_{1}$ in the inset of Fig. 5) propagating in the opposite direction as the incident beam. The curve in Fig. 5 displays the intensity of the first to AIP license or copyright, see http://ojps.aip.org/aplo/aplcr.jsp 


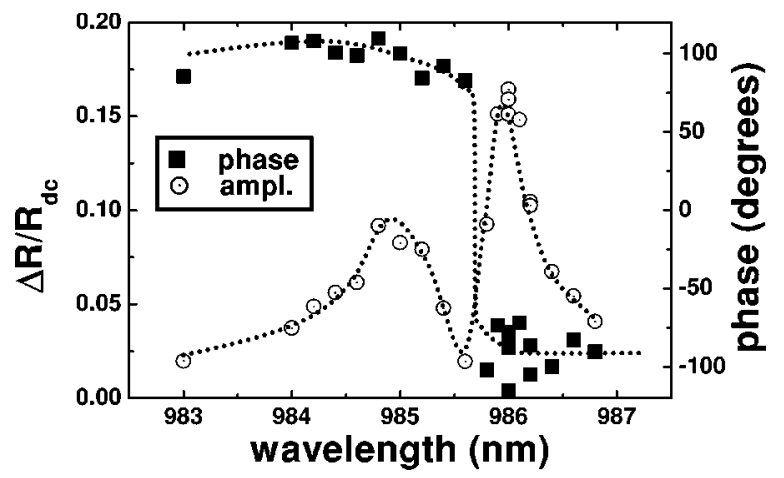

FIG. 4. Spectral dependence of the amplitude $\Delta R$ and phase of the ac reflectivity at the SAW frequency measured in the configuration of Fig. 3. $R_{d c}$ denotes the average reflectivity.

diffraction order $R_{1}$ recorded in this configuration in a resonator with $\lambda_{c}=919 \mathrm{~nm}$ and $Q=1300$, which reaches approximately $40 \%$ of the intensity of the incident beam for a SAW with $P_{l}=420 \mathrm{~W} / \mathrm{m}$.

The configuration of Fig. 5 turns the resonator into a versatile and compact Bragg cell, which can be operated as a frequency shifter, a switch, as well as a modulator. The frequency shifting action arises automatically from the fact that diffracted beam is shifted in frequency by $+\omega_{\text {SAW }}$ with respect to the incident one. Switching with a high on/off contrast is accomplished by simply turning the IDT on and off: the left inset of Fig. 5 shows optical micrographs of the sample recorded under these two conditions. Finally, the operation as a modulator is realized by modulating the rf voltage applied to the IDT with the information signal. Note that modulation action is also accompanied by a frequency shift $\omega_{\text {SAW }}$, a feature which can be explored for wavelength multiplexing applications.

In conclusion, we have demonstrated that strong acoustic and light fields in one-dimensional microscopic resonators lead to Brillouin scattering intensities comparable to the exciting one. The SAW-driven resonators based on this principle can be employed as photonic switches, frequency shifters, and modulators fabricated monolithically on GaAs. Finally, the same principle can easily be extended to control light flow in two- and three-dimensional PCs.

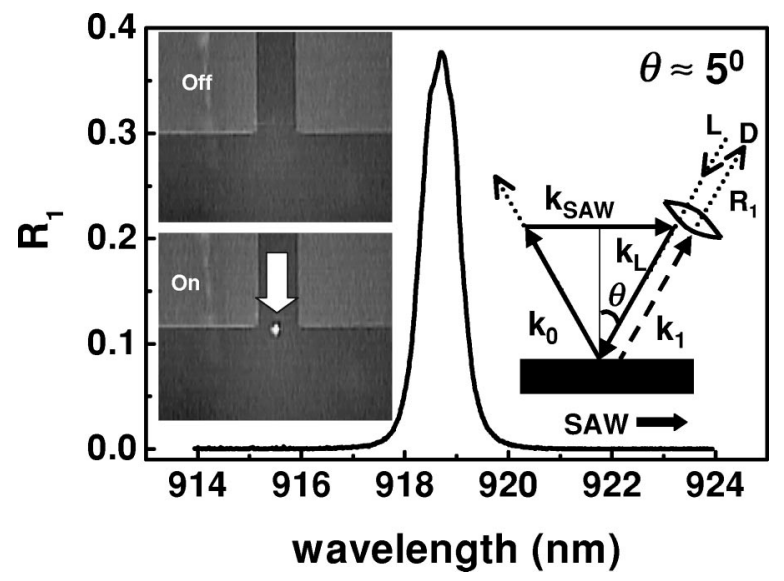

FIG. 5. Intensity of the first diffraction order $\left(R_{1}\right)$ for an angle of incidence $\theta \approx 5^{\circ}(\phi=0)$. The right inset displays the measurement setup. $k_{0}$ and $k_{1}$ denote the wave vectors of the zero- and first-order diffracted beams (not to scale). $k_{\text {SAW }}$ is the SAW wave vector. The left inset displays optical micrographs of the sample recorded with the rf power switched off (upper part) and on (lower part), showing the $R_{1}$ beam (bright dot). The light areas are the metallic pads of the IDT. The arrow indicates the propagation direction of the SAW beam.

The authors thank H. T. Grahn for comments and for a critical reading of the manuscript as well as S. Krauß and W. Seidel for the preparation of the samples. Support from the Deutsche Forschungsgemeinschaft (Project No. SA598/3-1) is gratefully acknowledged.

${ }^{1}$ K. Yoshino, Y. Shimoda, Y. Kawagishi, K. Nakayama, and M. Ozaki, Appl. Phys. Lett. 75, 932 (1999).

${ }^{2}$ K. Busch and S. John, Phys. Rev. Lett. 83, 967 (1999).

${ }^{3}$ S. W. Leonard, J. P. Mondia, H. M. van Driel, O. Toader, S. John, K. Busch, A. Birner, U. Gösele, and V. Lehmann, Phys. Rev. B 61, R2389 (2000).

${ }^{4}$ D. Kang, J. E. Maclennan, N. A. Clark, A. A. Zakhidov, and R. H. Baughman, Phys. Rev. Lett. 86, 4052 (2001).

${ }^{5}$ P. V. Santos and S. Krishnamurthy, European Patent Application 02003599.4 (2002).

${ }^{6}$ P. St. J. Russell, Phys. Rev. Lett. 56, 596 (1986).

${ }^{7}$ P. V. Santos, J. Appl. Phys. 89, 5060 (2001).

${ }^{8}$ A. Fainstein, B. Jusserand, and V. Thierry-Mieg, Phys. Rev. Lett. 75, 3764 (1995).

${ }^{9}$ M. Trigo, A. Bruchhausen, A. Fainstein, B. Jusserand, and V. ThierryMieg, Phys. Rev. Lett. 89, 227402 (2002). 